\title{
Synthesis of 2-Hydroxy-Ethyl Ester from Peanut Oil As A Bio-Additive for Diesel Fuel
}

\author{
Yulfi Zetra ${ }^{1}$ Laila Sukria ${ }^{1}$ R.Y Perry Burhan ${ }^{1,2,{ }^{*}}$ R. Arizal Firmansyah ${ }^{1,3}$ \\ ${ }^{1}$ Department of Chemistry, Faculty of Science and Data Analytics, Institut Teknologi Sepuluh Nopember, Surabaya, \\ 60111, Indonesia \\ ${ }^{2}$ Poilteknik Energi dan Mineral Akamigas, Jl Gajah Mada no 38, Cepu 58315, Indonesia \\ ${ }^{3}$ Department of Chemistry Education, Faculty of Science and Technology, Uin Walisongo, Semarang, 50185, \\ Indonesia \\ *Corresponding author.Email: pburhan@chem.its.ac.id
}

\begin{abstract}
Greenhouse gas emissions increase with the use of diesel fuel. The reduction of greenhouse gas emissions can be done by using the desulphuration method. However, low sulfur in diesel fuel causes low lubricity values. One of solutions to this problem is by adding a bio-additive compound. In this research, the synthesis of 2-hydroxyethyl ester compounds as a bio-additive obtained by the transesterification reaction of peanut oil with ethylene glycol and $\mathrm{K} 2 \mathrm{CO} 3$ catalyst will be carried out. The simple reflux is used with a molar ratio of ethylene glycol and triglycerides of 10: 1 with $\mathrm{K} 2 \mathrm{CO} 3$ of $9 \%$ mole of triglycerides. The conversion to the product was $77.47 \%$. Product characterization was carried out using Mass Spectrometry Gas Chromatography (GC-MS). Based on chromatograms, the total abundance of the 2hydroxyethyl ester is $54.69 \%$. This compound can be used as an alternative bio-additive lubricity improver.
\end{abstract}

Keywords: Bio-Additive, Transesterification, Peanut Oil, 2-Hydroxy-Ethyl Ester.

\section{INTRODUCTION}

Diesel fuel is the fuel used in diesel engines. The use of diesel engines is commonly used in various sectors of transportation, industrial equipment, power plants, and agricultural equipment [1]. However, the use of diesel fuel can cause environmental degradation with greenhouse gas emissions which include carbon monoxide (CO), carbon dioxide (CO2), nitrogen oxides (NO), nitrogen dioxide (NO2), and sulfur dioxide (SO2). The release of greenhouse gases into the atmosphere will trap a lot of heat which leads to environmental disasters such as the greenhouse effect, global warming, and acid rain [2].

The reduction of emission of $\mathrm{SO}_{2}$ as one of particulate matter can use the desulfurization method. However, this desulphurization method can actually reduce the lubricity properties of diesel fuel. Sulfur and aromatic compounds as natural lubricants for diesel fuel are also lost in the desulfurization process [3]. In fact, the lubricating properties of diesel fuel can be used as an engine lubricant to avoid friction in the engine which will accelerate wear on the engine [4]. Therefore, a lubricity- enhancing additive is needed to produce diesel fuel that is safe for health and the environment [5].

The additive as a lubricant enhancer can be influenced by the structure and functional groups of the molecule. One of them is that the presence of an alkyl ester chain from bio-additive synthesis products can increase the resulting lubricity value. The addition of 1methoxy-alkyl-ester synthesized from canola to diesel fuel has an effect on increasing the lubricity value which is marked by decreasing the value of wear scars (wear) [6]. The wear scar is the value of the wear diameter on the steel plate used in the lubricity test [7]. The high lubricity power is indicated by the small wear scar value. The addition of a hydroxyl group to the bioaditive substance molecule can increase the lubrication value. Knothe and Steidley (2005) [8] have reported a neat yield effect of $\mathrm{C} 3$ compounds with $\mathrm{OH}, \mathrm{NH} 2$, and $\mathrm{SH}$ groups indicating that oxygen increases lubrication more than nitrogen and sulfur characterized by a smaller wear scar value.

De Barros et al. [9] reported that the presence of unsaturated fatty acids higher than saturated fatty acids can be a factor in increasing diesel oil lubrication. 
Therefore, this research will carry out the transesterification reaction of triglycerides from peanut oil and ethylene glycol to produce 2-hydroxyethyl ester. Based on its structural composition, 2-hydroxyethyl ester can be used as a lubrication enhancing additive. Triglycerides from peanut oil are used as starting materials for the synthesis of lubrication-enhancing bioadditives because peanuts have a higher unsaturated fatty acid content than saturated fatty acids [10].

\section{MATERIALS AND METHOD}

\subsection{Materials}

The materials used in this study were peanut oil available in the market. The chemicals used are ethylene glycol, TLC Silica gel $60 \mathrm{~F}_{254}, \mathrm{~K}_{2} \mathrm{CO}_{3}, \mathrm{KOH}$, Methanol (Merck, Darmstadt, Germany). N-hexane (Fulltime, China). ethyl acetate and anhydrous sodium sulfate (Na2SO4) (Merck, Darmstadt, Germany). Distilled water, Whatman filter paper (GE Healthcare), litmus paper, aluminum foil, $10 \% \mathrm{HCl}$.

\subsection{Analysis of the Fatty Acid Composition of Peanut Oil}

Qualitative analysis of peanut oil and methanol transesterification products was accomplished using GCMS instrumentation (GCMS-QP2010 SE). The transesterification reaction method was adapted from Rashid et al. (2008) [11]. Peanut oil (triglycerides) and methanol reacted with a molar ratio of 1: 6 . The catalyst used is $\mathrm{KOH}$ at $1 \%$ of the mass of triglycerides (TG). TG is heated in a reflux flask to $60^{\circ} \mathrm{C}$. An amount of $\mathrm{KOH}$ in methanol is added to the reflux flask and the reaction time is conditioned to 2 hours and the temperature is maintained at $60^{\circ} \mathrm{C}$. Furthermore, the mixture is cooled at room temperature and left in a separating funnel overnight to form two layers. (water layer and organic layer). The organic layer is separated and the solvent evaporated using a rotary evaporator. The evaporated mixture was washed with distilled water, separated from the water layer and dried with anhydrous $\mathrm{Na} 2 \mathrm{SO} 4$, then filtered so that the product of the transesterification reaction of peanut oil was produced.

\subsection{Synthesis of 2-Hydroxyethyl Ester}

The synthesis procedure of 2-hydroxyethyl ester refers to a study adapted from Rezende et al. (2005) [5]. The molar ratio of triglycerides (TG) and ethylene glycol used was 1:10. A total of 20 grams of the TG sample is put into a three neck flask equipped with a reflux condenser, thermometer and magnetic stirrer heater. The TG sample was heated to $150^{\circ} \mathrm{C}$, ethylene glycol and $\mathrm{K} 2 \mathrm{CO} 3$ of $9 \%$ mol of $\mathrm{TG}$ were added to the three-neck flask and the reaction time was conditioned for 5 hours with constant stirring temperature at $500 \mathrm{rpm}$. After 5 hours, the mixture is cooled at room temperature and neutralized by adding a $10 \% \mathrm{HCl}$ solution to $\mathrm{pH} 7$. The neutral mixture is then extracted with ethyl acetate in a separating funnel. The product mixture is left for 12 hours to form 2 (two) phases, namely the water phase and the organic phase. The organic phase is separated, washed with water and the remaining water is dried over anhydrous sodium sulfate. Then the mixture is filtered and the remaining solvent is evaporated using a rotary evaporator to produce 2-hydroxyethyl ester (HEE) product.

\subsection{Monitoring of Synthesis Products Using Thin Layer Chromatography (TLC)}

The product of 2-hydroxyethyl ester (HEE), together with triglycerides, and ethylene glycol are dotted respectively on the TLC plate. Then the plate is inserted into the chamber and eluted with an eluent mixture (nhexane: ethyl acetate $2: 1$ ). The compound stains formed on the TLC plate were identified by the appearance of iodine stains to determine whether HEE synthesis products had been formed.

\subsection{Qualitative Analysis of 2-Hydroxyethyl Ester Product Using GCMS}

Qualitative analysis of the 2-hydroxyethyl ester (HEE) product was carried out using a gas chromatography instrument with a mass spectrometry detector (GC-MS QP-2020 NX) and equipped with a capillary column (Rtx-5MS) with a size $(30 \mathrm{~m} / 0.25$ mmID / 0,25 $\mu \mathrm{m}$ df). HEE sample preparation was carried out by diluting $0.1 \mathrm{~mL}$ of sample in $1 \mathrm{~mL}$ of $\mathrm{n}$ hexane solvent and injecting $1 \mu \mathrm{L}$ into the GCMS injector.

\section{RESULT AND DISCUSSION}

\subsection{Result}

\subsubsection{Identification of Fatty Acid Composition}

Identification of the fatty acid composition of peanut oil in the form of fatty acid methyl esters using the combined gas chromatography-mass spectroscopy (GCMS) method obtained the following chromatogram results: 


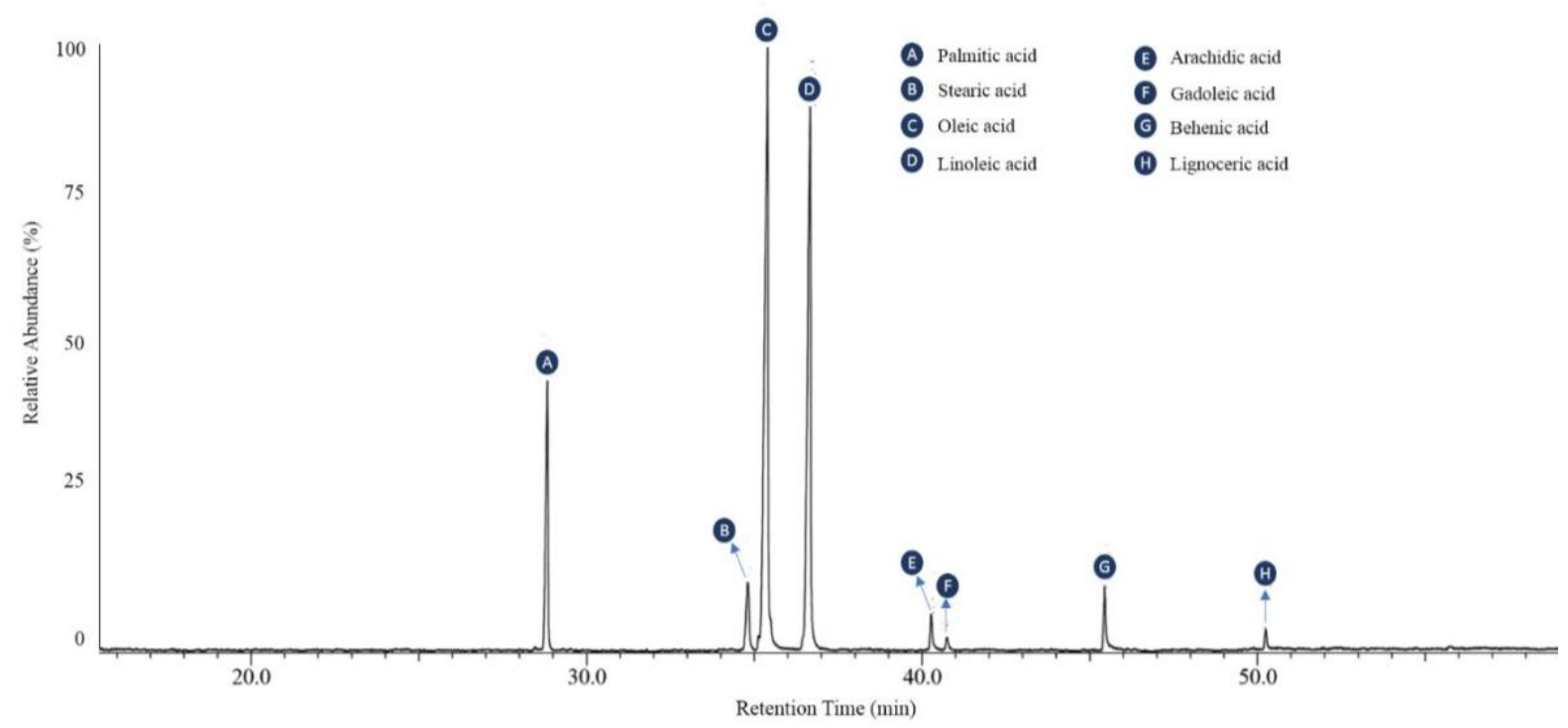

Figure 1 Chromatogram of product transesterification from peanut oil and methanol

\subsubsection{Synthesis of 2-Hydroxyethyl Ester}

Synthesis monitoring using TLC plates showed that the synthesis product in the form of 2-hydroxyethyl ester (HEE) has been formed as shown in Figure 2 below. Figure 2 shows that the position of product staining $(\mathrm{P})$ is not the same as TG and EG as an indicator of the formation of HEE products. The results displayed on the TLC plate are strengthened by the results of GCMS analysis in the form of a chromatogram as shown in Figure 3.

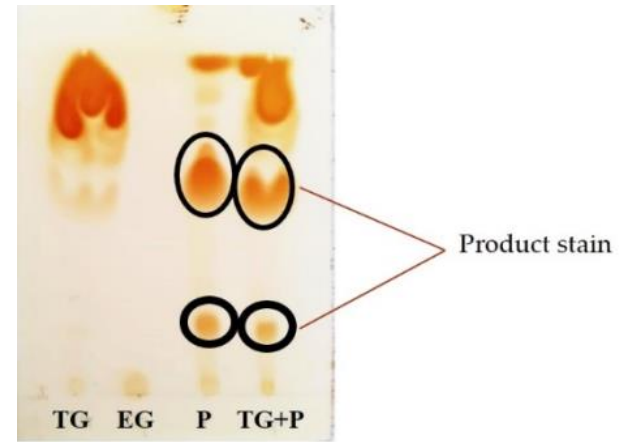

Figure 2 TLC of crude product of 2-hydroxyethyl ester. Abbreviations: $\mathrm{TG}=$ Triglyceride $; \mathrm{EG}=$ Ethylene Glycol; $\mathrm{P}=$ Product from synthesis

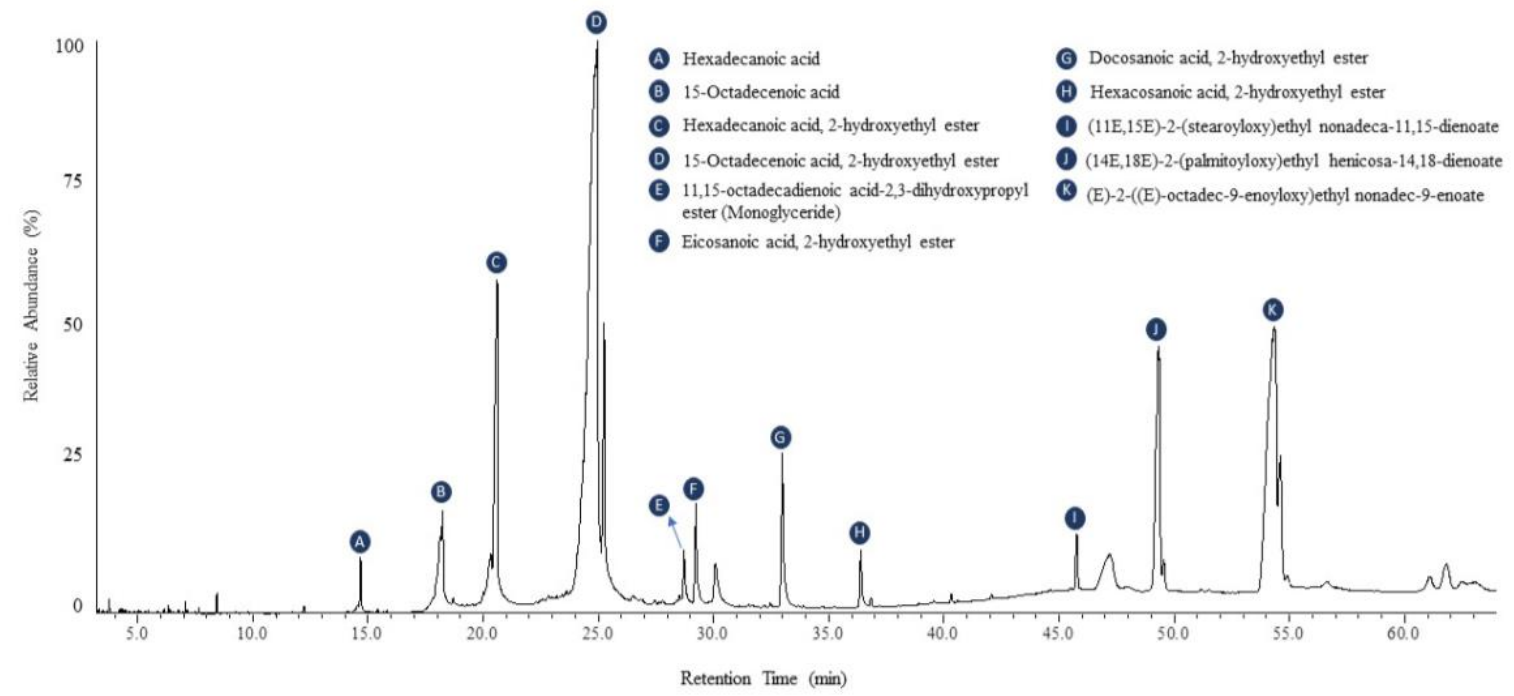

Figure 3 Chromatogram of HEE product 


\subsection{Discussion}

\subsubsection{Identification of Fatty Acid Composition}

The transesterification reaction of peanut oil samples with methanol aims to determine the fatty acid composition of the sample. The identification carried out based on the combined method of gas chromatographymass spectroscopy (GCMS) obtained results as shown in Table 1. Table 1 shows that oleic acid in peanut oil has the greatest abundance, namely $41.88 \%$ followed by linoleic acid at $34.67 \%$. These results are consistent with previous studies that the content of oleic acid and linoleic acid, which are unsaturated fatty acids in peanut oil, were respectively $35.6-58.3 \%$ and $20.9-43.2 \%$ [12]. The amount of unsaturated fatty acid content in peanut oil has the potential to be used as a lubricant-enhancing bioadditive. This is also in accordance with the research of De Barros et al., 2017 [9] where the presence of unsaturated fatty acid groups in oleic acid and linoleic acid has succeeded in increasing the lubricity value of diesel fuel which is characterized by reduced friction value on metals.

Table 1. Composition of fatty acid from sample from analysis of GCMS

\begin{tabular}{llll}
\hline No. & RT (minute) & Composition of Fatty Acid & \%Abundance \\
\hline A & 28.8 & Palmitic acid & 12.35 \\
B & 34.8 & Stearic acid & 4.28 \\
C & 35.4 & Oleic acid & 41.88 \\
D & 36.6 & Linoleic acid & 34.67 \\
E & 40.2 & Arachidic acid & 1.91 \\
F & 40.7 & Gadoleic acid & 0.82 \\
G & 45.4 & Behenic acid & 3.19 \\
H & 50.2 & Lignoceric acid & 0.90 \\
\hline
\end{tabular}

\subsubsection{Synthesis of 2-Hydroxyethyl Ester}

Synthesis of 2-hydroxyethyl ester (HEE) was carried out by transesterification of triglycerides from peanut oil and ethylene glycol using K2CO3 as a catalyst. Product monitoring was carried out by thin layer chromatography with n-hexane: ethyl acetate 2: 1 eluent as shown in Figure 1. The TLC results showed that the product was formed which was marked by the appearance of new stains identified as product (P). Synthesis follows the following reaction flow.

The 2-hydroxyethyl ester products identified based on mass spectrum data analysis are shown in Table 2 . The 2-hydroxyethyl ester products formed include various different hydrocarbon chains and there are still side products in the form of fatty acids. The highest chromatogram peak is shown at the fourth peak at the retention time of 24.9 minutes as seen in Figure 3. The four (D) peaks are 15-octadecenoic acid, 2-hydroxyethyl ester with an abundance of $41.34 \%$. The highest abundance yields of hydroxy ethyl ester products correspond to the highest type of fatty acid contained in peanut oil, namely oleic acid (15-octadecanoic acid) [12].

The second highest abundance of $21.62 \%$ was indicated by a peak of 11 with a retention time of 54.3 minutes. This compound has been identified as a byproduct of Ethylene Glycol Diester ((E) -2 - ((E) octadec-9-enoyloxy) ethyl nonadec-9-enoate). Several studies that have been published by previous researchers also stated that the presence of ethylene glycol diester (EGDE) in synthetic products can actually act as biolubricants [13]. However, the research that has been done here shows that the total of all HEE products produced still shows the majority compared to other synthetic products. The total abundance value obtained was $54.69 \%$ while the total product conversion formed was $77.47 \%$.

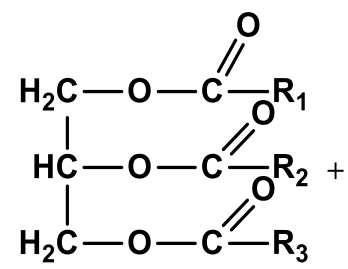

Triglycerides

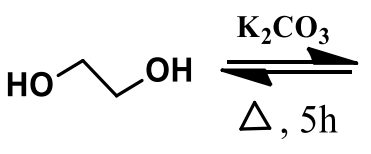

HO<smiles>[R]C(=O)OCCC</smiles><smiles>[R]C(=O)OCCOC([R])=O</smiles>

Ethylene Glycol Diester (EGDE)

Figure 4 Transesterification reaction of triglycerides and ethylene glycol 
Table 2. Composition of compounds in the crude product

\begin{tabular}{lccl} 
No. & RT (minute) & \%Abundance & \multicolumn{1}{c}{ Compounds } \\
\hline A & 14.6 & 1.12 & Hexadecanoic acid \\
B & 18.2 & 0.88 & 15-Octadecenoic acid \\
C & 20.6 & 7.26 & Hexadecanoic acid, 2-hydroxyethyl ester \\
D & 24.9 & 41.34 & 15-Octadecenoic acid, 2-hydroxyethyl ester \\
E & 28.7 & 0.82 & 11,15-octadecadienoic acid-2,3-dihydroxypropyl ester (Monoglyceride) \\
F & 29.2 & 1.85 & Eicosanoic acid, 2-hydroxyethyl ester \\
G & 32.9 & 3.27 & Docosanoic acid, 2-hydroxyethyl ester \\
H & 36.3 & 0.97 & Hexacosanoic acid, 2-hydroxyethyl ester \\
I & 45.7 & 0.93 & (11E,15E)-2-(stearoyloxy)ethyl nonadeca-11,15-dienoate \\
J & 49.3 & 8.99 & (14E,18E)-2-(palmitoyloxy)ethyl henicosa-14,18-dienoate \\
K & 54.3 & 21.62 & (E)-2-((E)-octadec-9-enoyloxy)ethyl nonadec-9-enoate \\
\hline
\end{tabular}

\section{CONCLUSION}

The 2-hydroxyethyl ester (HEE) compound as a bioadditive to increase diesel lubrication was successfully synthesized through the transesterification reaction of peanut oil and ethylene glycol with $\mathrm{K} 2 \mathrm{CO} 3$ catalyst. This study resulted in a product conversion of $77.47 \%$ with the total abundance of HEE obtained through GCMS analysis of $54.69 \%$.

\section{ACKNOWLEDGMENTS}

The authors fully acknowledged the Ministry of Energy and Mineral Resources who has given financial support. PEM-Akamigas Cepu, Central of Java and Institut Teknologi Sepuluh Nopember Surabaya, East of Java, who has given the technical support to carry out this research and to write this journal.

\section{REFERENCES}

[1] Le Ning, Qimeng Duan, Zhanming Chen, Hailiang Kou, Bing Liu, Bo Yang, Ke Zeng., "A comparative study on the combustion and emissions of a nonroad common rail diesel engine fueled with primary alcohol fuels (methanol, ethanol, and nbutanol)/diesel dual fuel," Fuel, vol. 266, p. 117034, Apr. 2020, doi: 10.1016/j.fuel.2020.117034.

[2] K. T. Tan and K. T. Lee, "Biodiesel Production in Supercritical Fluids," in Biofuels: Alternative Feedstocks and Conversion Processes for the Production of Liquid and Gaseous Biofuels, Elsevier, 2019, pp. 523-538.

[3] P. Y. Hsieh, J. A. Widegren, A. J. Slifka, A. J. Hagen, and R. A. L. Rorrer, "Direct Measurement of Trace Polycyclic Aromatic Hydrocarbons in Diesel Fuel with ${ }^{1} \mathrm{H}$ and ${ }^{13} \mathrm{C}$ NMR Spectroscopy: Effect of PAH Content on Fuel Lubricity," Energy Fuels, vol. 29, no. 7, pp. 4289-4297, Jul. 2015, doi: 10.1021/acs.energyfuels.5b01193.
[4] G. Karmakar, P. Ghosh, and B. Sharma, "Chemically Modifying Vegetable Oils to Prepare Green Lubricants," Lubricants, vol. 5, no. 4, p. 44, Nov. 2017, doi: 10.3390/lubricants5040044.

[5] M. J. C. Rezende, C. R. Perruso, D. de A. Azevedo, and A. C. Pinto, "Characterization of lubricity improver additive in diesel by gas chromatographymass spectrometry," J. Chromatogr. A, vol. 1063, no. 1-2, pp. 211-215, Jan. 2005, doi: 10.1016/j.chroma.2004.12.015.

[6] S. Wang, J. Shen, and M. J. T. Reaney, "LubricityEnhancing Low-Temperature Diesel Fuel Additives," J. Am. Oil Chem. Soc., vol. 89, no. 3, pp. 513-522, Mar. 2012, doi: 10.1007/s11746-0111932-7.

[7] S. Syahrullail, N. Nuraliza, M. I. Izhan, M. K. A. Hamid, and D. M. Razaka, "Wear Characteristic of Palm Olein as Lubricant in Different Rotating Speed," Procedia Eng., vol. 68, pp. 158-165, 2013, doi: 10.1016/j.proeng.2013.12.162.

[8] G. Knothe and K. R. Steidley, "Lubricity of Components of Biodiesel and Petrodiesel. The Origin of Biodiesel Lubricity ${ }^{\dagger}, "$ Energy Fuels, vol. 19, no. 3, pp. 1192-1200, May 2005, doi: 10.1021/ef049684c.

[9] M. I. De Barros Bouchet Jean Michel Martin, a Cyrielle Forest,a Thierry le Mogne, a Micha"el Mazarin,b Jos'e Avila,c Maria C. Asensioc and Gregory L. Fisher., "Tribochemistry of unsaturated fatty acids as friction modifiers in (bio)diesel fuel," RSC Adv., vol. 7, no. 53, pp. 33120-33131, 2017, doi: 10.1039/C7RA02926H.

[10] M. Taha, H. Yousef, S. EL-Behery, and H. Mostafa, "Fatty acids and chemical composition of peanut (Arachis hypogaea L.)," Al-Azhar J. Agric. Res., vol. 44, no. 1, pp. 119-127, Jun. 2019, doi: 10.21608/ajar.2019.59735. 
[11] U. Rashid, F. Anwar, B. R. Moser, and S. Ashraf, "Production of sunflower oil methyl esters by optimized alkali-catalyzed methanolysis," Biomass Bioenergy, vol. 32, no. 12, pp. 1202-1205, Dec. 2008, doi: 10.1016/j.biombioe.2008.03.001.

[12] M. E. Carrnn and A. A. Carelli, "Peanut oil: Compositional data," Eur J Lipid Sci Technol, p. 11, 2010.
[13] N. K. Attia, S. A. El-Mekkawi, O. A. Elardy, and E. A. Abdelkader, "Chemical and rheological assessment of produced biolubricants from different vegetable oils," Fuel, vol. 271, p. 117578, Jul. 2020, doi: 10.1016/j.fuel.2020.117578. 